\title{
Digital Technology in Skin Cancer Prevention and Early Detection
}

Monika Janda ${ }^{1}$, PhD; Carina V Silva ${ }^{1}$, MPH; Caitlin Horsham ${ }^{1}$, MSc; Craig Sinclair ${ }^{2}$, MPPM; Montana O'Hara ${ }^{1}$, BHlthSci; Peter Baade ${ }^{3}$, PhD; H Peter Soyer ${ }^{4}$, MD

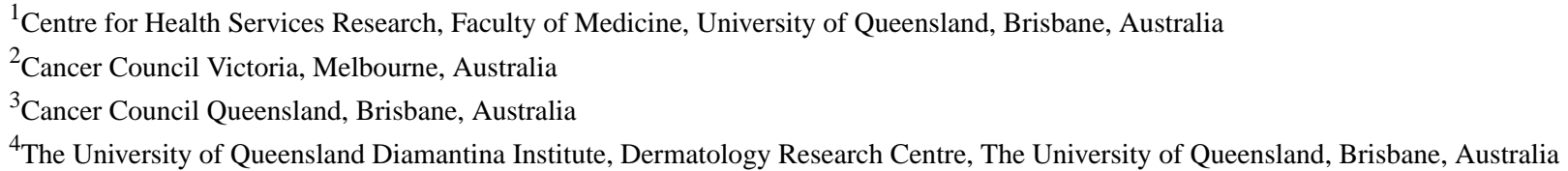

\section{Corresponding Author:}

Monika Janda, $\mathrm{PhD}$

Centre for Health Services Research

Faculty of Medicine

University of Queensland

34 Cornwall St

Brisbane, 4102

Australia

Phone: 61731764569

Email: m.janda@uq.edu.au

\section{Abstract}

Background: Mobile teledermatology is increasingly being used in clinical practice and offers the opportunity to counsel the general public about sun protection and skin cancer early detection. Growing evidence suggests that SMS text messaging interventions are an effective way to reach a large number of people and promote sun protection behaviors. Many medical practices already have SMS text message systems in place to communicate with patients, especially for appointment reminders and information. However, could we use these systems for even better outcomes? If so, how?

Objective: This presentation will outline the results of the SunText study, a theory-based SMS text messaging intervention designed to evaluate how often and in what way we could communicate with people at risk of skin cancer to have a beneficial effect on sun protection behaviors, sunburn, and participant engagement.

Methods: The SunText study was conducted between February-July 2019 in Queensland, Australia. Volunteer participants aged 18 to 40 years were randomized to 4 different intervention schedules using a Latin square design. The schedules included personalized or interactive messages with constant frequency and personalized and interactive messages with either increasing or decreasing frequency. Outcomes measured were reduction in sunburn and engagement with interactive messages, defined as responding to messages by return text.

Results: Compared to baseline, the self-reported sun protection habits index was significantly higher in all 4 interventions $(P<.01)$. Overall, sunburn rates decreased from baseline to the end of the intervention ( $40.3 \%$ to $7.0 \%)$, and remained significantly below baseline levels $(23.5 \%)$ at the 6-month follow-up $(P<.01)$. All 4 interventions achieved reductions in sunburn rates $(18 \%-48 \%$ reduction) during the intervention period. The overall engagement rate with interactive messages was $71 \%$. The intervention involving interactive messages with constant frequency achieved the highest engagement rate. The intervention with personalized and interactive messages with increasing frequency had the lowest engagement rate.

Conclusions: This study adds to the evidence that text messages targeting sun protection are effective in improving sun protection behaviors and reducing sunburn. Results also suggest higher engagement with constant or decreasing message frequency. Although many clinics already use SMS text messaging for scheduling, this presentation may encourage its extended use to raise awareness of sun protection. Interactive messages could also be integrated into sun protection mobile health apps, and provide an opportunity for engaging in health promotion content.

Acknowledgments: This study was funded by a research grant from the Harry J Lloyd Charitable Trust.

Conflicts of Interest: HPS is a shareholder of MoleMap NZ Limited and e-derm consult GmbH, and undertakes regular teledermatological reporting for both companies. HPS is a Medical Consultant for Canfield Scientific Inc, MoleMap Australia Pty Ltd, Blaze Bioscience Inc, Revenio Research Oy and a Medical Advisor for First Derm. All other authors declare no conflicts of interest. 
(iproc 2022;8(1):e36908) doi: $10.2196 / 36908$

\section{KEYWORDS}

skin cancer; prevention; mHealth; text-delivered intervention; engagement

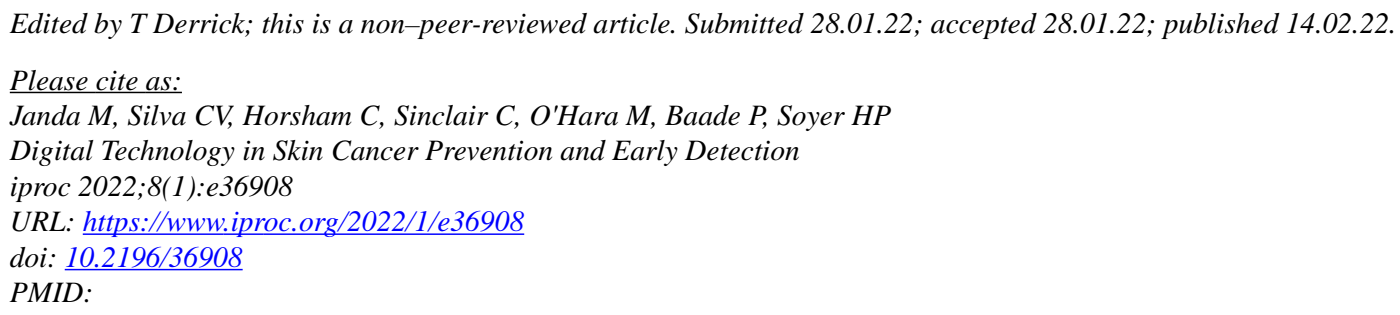

CMonika Janda, Carina V Silva, Caitlin Horsham, Craig Sinclair, Montana O'Hara, Peter Baade, H Peter Soyer. Originally published in Iproceedings (https://www.iproc.org), 14.02.2022. This is an open-access article distributed under the terms of the Creative Commons Attribution License (https://creativecommons.org/licenses/by/4.0/), which permits unrestricted use, distribution, and reproduction in any medium, provided the original work, first published in Iproceedings, is properly cited. The complete bibliographic information, a link to the original publication on https://www.iproc.org/, as well as this copyright and license information must be included. 